\title{
Preface
}

\section{Physiopathology of Vascular Risk Factors in Alzheimer's Disease}

\author{
Jack C. de la Torre* \\ Professor of Psychology (Adjunct), University of Texas, Austin, USA
}

When one thinks of vascular risk factors to Alzheimer's disease (VRF-AD), several things may come to mind. First, what are VRF-AD and why are they important? Second, why or what makes them a pathological risk to Alzheimer's disease (AD)? Third, how can they be identified or diagnosed? Fourth, what interventions if any, can be used to manage VRF-AD and could they help reduce or reverse the prevalence of cognitive decline and AD?

This special issue of the Journal of Alzheimer's Disease titled "Physiopathology of Vascular Risk Factors in Alzheimer's Disease" is dedicated to these four questions and many others which relate to their profile, pathophysiology, diagnosis, and intervention. A panel of international experts from 16 countries pooled their knowledge to provide clarity and insight into VRF-AD, one of the fastest growing fields in AD research.

VRF-AD were rarely if ever mentioned in the medical literature prior to 1997 . That year, several prospective epidemiological studies discovered what turned out to be a breakthrough in our limited understanding of AD. The studies reported how specific maladies that directly affected the cerebral vasculature could lead to the development of cognitive decline and $\mathrm{AD}$ onset. As more studies began evaluating large populations of middle age and elderly individuals with hypertension, atherosclerosis, diabetes type 2 , hyperlipidemia, and heart disease, it became clear that a

\footnotetext{
${ }^{*}$ Correspondence to: Jack C. de la Torre, MD, PhD, Professor of Psychology (Adjunct), University of Texas, Austin, TX 78712, USA. Tel.: +760 703 0585; Fax: +623 876 5378; E-mail: jcdelatorre@comcast.net.
}

significant association between these disorders and an increased risk of $\mathrm{AD}$ existed. An avalanche of research surveys soon followed as newly described VRF-AD were identified. Moreover, parallel studies in neuropathology, cognitive function, neuroimaging, and clinical trials systematically began to blur the nosological differences between AD and vascular dementia.

Key research targets in this special issue include examination of pathological, biochemical, physiologi$\mathrm{cal}$, and metabolic processes that contribute to AD risk and ways that may delay or reverse these age-related abnormalities.

What has VRF-AD research taught us so far? Some of the answers are contained in the current chapters of this issue. But one consistent finding rises above all others. It is that VRF-AD offer the possibility of markedly reducing incident dementia by early identification and appropriate medical management of these presumed precursors of cognitive deterioration and dementia. Will it be possible to eradicate AD altogether with this approach? Hopefully so, but not for a long time. Nevertheless, improved understanding coupled with preventive strategies to VRF-AD could be a monumental step forward in reducing the worldwide prevalence of dementia. According to the World Alzheimer Report 2010, dementia will rise from a current 35 million to 65 million people by the year 2030 . This is a staggering and grim statistic because AD prevalence is doubling every 20 years. To deflect this estimate, the model of lung cancer deaths in the US, of which $88 \%$ are caused by cigarette smoking, could be adopted. Aggressive anti-smoking campaign ads that began in earnest about 
20 years ago have been able to convince smokers to quit, a fact that has saved about a million lung cancer deaths every year in the U.S. alone. That is an investment that could really pay off if AD risks were reduced substantially, not only from a fiscal viewpoint but in delaying ill-health and a dreaded brain disorder among the elderly. Since the presence of VRF-AD is not an absolute pathway to dementia, it may be as important, if not more so, to study how or why individuals who are cognitively normal but show these risks are able to avoid dementia.

This task of reducing incident $\mathrm{AD}$ by managing or treating VRF-AD is in its infancy but if it is to succeed, education of primary care physicians and specialists will be as important as educating the public. Primary care providers will be the first line of defense because they are likely to first see patients who harbor
AD risk factors. Simple laboratory testing can establish the presence of most major VRF-AD. Referral from primary care physicians to the second line of defense, specialists such as neurologists cardiologists, dieticians, psychologists, and others trained in gerontological health, can implement a plan to control the preclinical morbidity of VRF-AD, ideally before any symptoms of cognitive dysfunction appear and certainly before the consequences of VRF-AD lead to ill health and cognitive meltdown.

Reducing AD prevalence by focusing right now on VRF-AD even with our limited technology is not a simple or easy task. But the task must begin somewhere and without delay because time is running out for millions of people whose destiny with dementia may start sooner than later. 